\title{
Consumption of a plant sterol-based spread derived from rice bran oil is effective at reducing plasma lipid levels in mildly hypercholesterolaemic individuals
}

\author{
Sarah Eady ${ }^{1 *}$, Alison Wallace ${ }^{1}$, Jinny Willis ${ }^{2}$, Russell Scott ${ }^{2}$ and Chris Frampton ${ }^{3}$ \\ ${ }^{1}$ The New Zealand Institute for Plant and Food Research Limited, Private Bag 4704, Christchurch, New Zealand \\ ${ }^{2}$ Lipid and Diabetes Research Group, Christchurch Hospital, Christchurch, New Zealand \\ ${ }^{3}$ Christchurch School of Medicine and Health Sciences, PO Box 4345, Christchurch, New Zealand
}

(Received 25 May 2010 - Revised 18 November 2010 - Accepted 1 December 2010 - First published online 15 February 2011)

\section{Abstract}

To establish the effectiveness of a new phytosterol-containing spread derived from rice bran oil (RBO), a randomised, double-blind, crossover human clinical trial was conducted over 12 weeks. A total of eighty mildly hypercholesterolaemic (total blood cholesterol level $\geq 5$ and $\leq 7.5 \mathrm{mmol} / \mathrm{l}$ with a serum TAG level of $\leq 4.5 \mathrm{mmol} / \mathrm{l}$ ) individuals were randomised into two groups ( $n$ 40). Group 1 consumed spread only daily for 4 weeks. They were randomised to consume $20 \mathrm{~g}$ RBO spread (RBOS), $20 \mathrm{~g}$ standard spread (SS) or $20 \mathrm{~g}$ phytosterol-enriched spread (PS). After a 4-week period, individuals changed to the next randomised treatment until all three treatments had been consumed. Group 2 consumed spread plus oil daily for 4 weeks. They consumed $20 \mathrm{~g}$ RBOS plus $30 \mathrm{ml}$ RBO, $20 \mathrm{~g}$ SS plus $30 \mathrm{ml}$ sunflower oil or $20 \mathrm{~g}$ RBOS. Blood samples were collected for the analysis of lipid parameters, and $3 \mathrm{~d}$ diet records were collected. Compared with SS, RBOS significantly reduced total cholesterol by $2 \cdot 2 \%(P=0.045)$, total cholesterol:HDL by $4 \cdot 1 \%(P=0 \cdot 005)$ and LDL-cholesterol by $3 \cdot 5 \%$ $(P=0.016)$, but was not as effective overall as PS, which reduced total cholesterol by $4 \cdot 4 \%(P=0 \cdot 001)$, total cholesterol:HDL by $3 \cdot 4 \%$ $(P=0.014)$ and LDL-cholesterol by $5.6 \%(P=0.001)$. In group 2 , the addition of RBO to the RBOS produced no differences in cholesterol levels. These results confirm that RBOS is effective in lowering serum cholesterol when consumed as part of a normal diet.

\section{Key words: Plant sterols: Cholesterol: Lipoproteins}

LDL-cholesterol (LDL-C) is now identified as the primary target for the clinical management of CHD risk, and forms the basis of the US National Cholesterol Education Programme ${ }^{(1)}$. Dietary incorporation of plant sterols is a recommended therapeutic approach within the National Cholesterol Education Programme to control circulating $\mathrm{LDL}^{(2)}$. These naturally occurring plant compounds are structurally and functionally similar to cholesterol, allowing them to displace cholesterol in the mixed micelle and reduce intestinal cholesterol absorption, although the exact mechanism of action is not yet elucidated $^{(3)}$. Recent research has suggested that plant sterols may produce up to a $15 \%$ reduction in plasma LDL-C at a dosage of $2 \mathrm{~g} / \mathrm{d}$ without any reported side effects ${ }^{(4)}$. The most abundant plant sterols are the 4-desmethyl sterols comprising sitosterol, stigmasterol and campesterol, and levels in the typical Western diet are thought to be approximately 100-300 mg sterols and 20-50 mg stanol (the hydrogenated form of sterols)/d; consequently, some form of supplementation is required to provide the recommended daily dosage $e^{(4,5)}$. In the 'free' unesterified form, plant sterols are hydrophobic and have limited solubility; thus, plant sterols currently incorporated into foods are esterified to unsaturated fatty acids (creating sterol esters), increasing lipid solubility and the ability to incorporate these compounds into fatbased foods. Plant sterol-enriched spreads are a popular vehicle for the delivery of these compounds, and functional foods of this nature have been available to consumers worldwide since $1995^{(6-8)}$. They have been widely studied for their influence on LDL-C lowering, producing consistent reductions of 9 and $15 \%$ without any reported side effects ${ }^{(7,9,10)}$. Vegetable oils such as maize, soyabean, safflower, sunflower and rapeseed are most commonly used as a base of these functional plant sterol-based spreads; however, rice bran oil (RBO) is now emerging as an effective alternative for vegetable oils both in the manufacture of these spreads and as an everyday cooking oil ${ }^{(11)}$. Rice bran is a by-product of

Abbreviations: HDL-C, HDL-cholesterol; LDL-C, LDL-cholesterol; PS, phytosterol-enriched spread; RBO, rice bran oil; RBOS, rice bran oil spread; SS, standard spread; TC, total cholesterol. 
the rice milling industry, separating from the white portion of the rice in the polishing process. Human consumption has been traditionally limited because of the bran's instability and the rapid onset of rancidity produced by a high lipase activity in the bran causing deterioration of the lipids ${ }^{(12)}$. However, improved oil extraction methods have reduced this problem, and in Asian countries such as Japan, China, Korea and Thailand, RBO consumption has increased ${ }^{(13)}$. Studies have verified that RBO demonstrates the ability to lower LDL-C levels to an equal if not better standard than other vegetable oils, and this effect is largely attributed to the unusually high levels of unsaponifiable material found in $\mathrm{RBO}^{(14)}$. This is composed of plant sterols, triterpene alcohols, ferulic acid esters ( $\gamma$-oryzanol) and vitamin $\mathrm{E}$ isomers (tocopherols and tocotrienols). In addition, RBO contains up to $20 \%$ SFA and equal amounts of MUFA and PUFA ${ }^{(11)}$. Collectively, these components confer reputed health benefits to $\mathrm{RBO}$ that include antioxidant potential, improved lipid metabolism, anti-cancer action, anti-atherogenic action and improved immune function ${ }^{(15)}$. The oil is also attractive to consumers, having a mild flavour and high smoking point, making it suitable for use in many cooking methods and importantly, no adverse effects have been reported. The potential availability of this product is high, as rice is a major cereal crop in many countries; thus, interest in this product is increasing ${ }^{(16)}$.

The aim of the present study was to determine the effect of including a spread based on RBO that may have LDLC-lowering ability, into the daily diet. We aimed to establish its potential compared with standard polyunsaturated spreads and other commercially available plant sterol-containing spreads. Additionally, we examined whether any cholesterollowering activity could be enhanced by including a supplementary serving of the corresponding base oil of the spreads to the diet, as we envisaged that this may mimic consumer behaviour when aiming to achieve maximum benefit from the plant sterol-containing product.

\section{Methods}

\section{Participants}

Participants were recruited through advertisements and articles in the local Christchurch (New Zealand) newspapers. Eligible participants were healthy and aged between 30 and 65 years with a BMI of $<35 \mathrm{~kg} / \mathrm{m}^{2}$. Total cholesterol (TC) levels were required to be $\geq 5$ and $\leq 7.5 \mathrm{mmol} / \mathrm{l}$, with serum TAG levels below $4.5 \mathrm{mmol} / \mathrm{l}$. Participants were required to have normal glucose tolerance with fasting blood glucose levels of $\leq 6.1 \mathrm{mmol} / 1$ and were not eligible if they were smokers, had a history of diabetes or were taking lipid-lowering medication or other medication likely to affect lipid metabolism. Other exclusion criteria included renal, hepatic, cardiovascular, endocrine, gastrointestinal or other systemic disease; any known blood-borne disease, untreated hypertension, pregnancy, history of substance abuse including alcohol abuse, extreme dietary habits or food allergies and extreme exercise regimens. Participant demographic characteristics are shown in Table 3. The present study was conducted according to the guidelines laid down in the Declaration of Helsinki, and all procedures involving human subjects were approved by the Canterbury Upper South A Ethics Committee. Written informed consent was obtained from all subjects.

\section{Experimental design}

The study was a randomised, double-blind, cross-over trial of two dietary interventions based on consuming plant sterolbased spread and oil products. A total of eighty individuals were randomly assigned to one of two groups ( $n$ 40).

Group 1 was asked to substitute RBO spread (RBOS) for their usual spread, while group 2 was asked to include both spread and the corresponding oil on which the spread was based. All participants were asked to exclude any plant sterol-containing products from their diet for at least 3 weeks before the start of the trial. This included avoiding any commercially available plant sterol-based spread plus RBO and any products based on this oil. Participants were given one-on-one interviews at the point of screening to discuss the dietary implications of this requirement. Baseline measurements were taken before allocation of the first intervention. Participants were asked to complete an estimated $3 \mathrm{~d}$ diet record of their usual dietary pattern and were asked to use three non-consecutive days including one weekend/ non-working day. They were also given a colour picture booklet to help their estimation of food portion sizes. Fasting venous blood samples were collected on two occasions on non-consecutive days for the determination of blood glucose, TC, LDL-C, HDL-cholesterol (HDL-C), TC:HDL-C and TAG. Height, weight and blood pressure measurements were also collected. With the exception of blood glucose, individual measurements of lipid levels, blood pressure, height and weight were repeated at the end of each intervention stage.

Group 1 ( $n$ 40) was then assigned to the spread-only group. The participants were randomised by an independent statistician using a Microsoft EXCEL randomisation procedure to consume either $20 \mathrm{~g}$ RBOS daily for 4 weeks, $20 \mathrm{~g}$ standard spread (SS) daily for 4 weeks or $20 \mathrm{~g}$ phytosterol-enriched spread (PS) daily for 4 weeks. At the end of each 4 -week period, individuals changed to the next treatment within their group. No washout period was included because the length of each treatment period was 4 weeks, which provided enough time for the stabilisation of lipid levels even after a change in dietary intake. Phytosterol levels delivered in the $20 \mathrm{~g}$ amounts were as follows: RBOS, $118 \mathrm{mg}$ phytosterol and $30 \mathrm{mg}$ - -oryzanol; PS, $1600 \mathrm{mg}$ phytosterol; SS, $0 \mathrm{mg}$ phytosterol.

Group 2 ( $n$ 40) was allocated to the spread plus oil group and consumed $20 \mathrm{~g}$ RBOS plus $30 \mathrm{ml}$ RBO daily for 4 weeks, $20 \mathrm{~g}$ SS plus $30 \mathrm{ml}$ sunflower oil daily for 4 weeks or $20 \mathrm{~g}$ RBOS daily for 4 weeks. At the end of each 4 -week period, individuals changed to the next treatment within their group. Phytosterol amounts delivered in these amounts were as follows: RBOS, $118 \mathrm{mg}$ phytosterol and $30 \mathrm{mg} \gamma$-oryzanol; RBO, $222 \mathrm{mg}$ phytoterol and $150 \mathrm{mg} \gamma$-oryzanol. Fig. 1 shows a schematic of the trial design. 
Participants were required to use the trial products as a replacement for any spread/spread products already included in their everyday diet and were asked not to exceed the specified amounts. Participants were given identical sets of measuring spoons to measure out the correct amount of spread and oils each day. In all other aspects, individuals were required to maintain their normal dietary intake patterns and encouraged to keep their lifestyle, such as levels of physical activity, constant throughout the study. At each visit to the clinic, participants were given one-on-one interviews and asked to highlight any concerns relating to the study and any changes to their normal daily routines. Individuals were asked to report any sickness or commencement of new medication and changes to physical activity levels.

From each individual, four $3 \mathrm{~d}$ diet records and eight blood samples were collected. Blood pressure, height and weight measurements were collected on every clinic visit, following each intervention period, totalling four measurements in all. There were no washout periods during the study.

\section{Study products}

The composition of the spreads and oils used in the present study is shown in Tables 1 and 2, respectively. The RBOS and oil were manufactured by Old Fashioned Foods Limited,
Auckland, New Zealand, and the SS and PS were manufactured by Unilever, Minto, NSW, Australia. Sunflower oil was manufactured by Tasti Products Limited, Auckland, New Zealand.

All products were packaged in identical unmarked tubs or oil bottles and given unique identifier codes by an independent individual to ensure blinding to both researchers and participants. The codes were revealed after the completion of the trial.

\section{Blood collection and analysis}

Fasting blood samples were collected into tubes containing heparin for lipid analysis and tubes containing fluoride/oxalate for glucose analysis. Following collection, blood samples were centrifuged at $3000 \mathrm{~g}$ for $10 \mathrm{~min}$, and serum samples were removed and frozen at $-20^{\circ} \mathrm{C}$ for subsequent analysis at Canterbury Health Laboratories (Christchurch, New Zealand). TC was measured using enzymatic cholesterol oxidase and Abbott reagent on an Aeroset/c8000 analyser. HDL-C was analysed using enzymatic Roche reagent on an Aeroset/ c8000 analyser. TAG was measured using enzymatic hydrolysis of TAG with Abbott reagent on an Aeroset/c8000 analyser. LDL-C was calculated by difference using the Friedewald equation $^{(17)}$. Calculation of LDL-cholesterol is an approximation and is invalid in the presence of VLDL. Therefore,

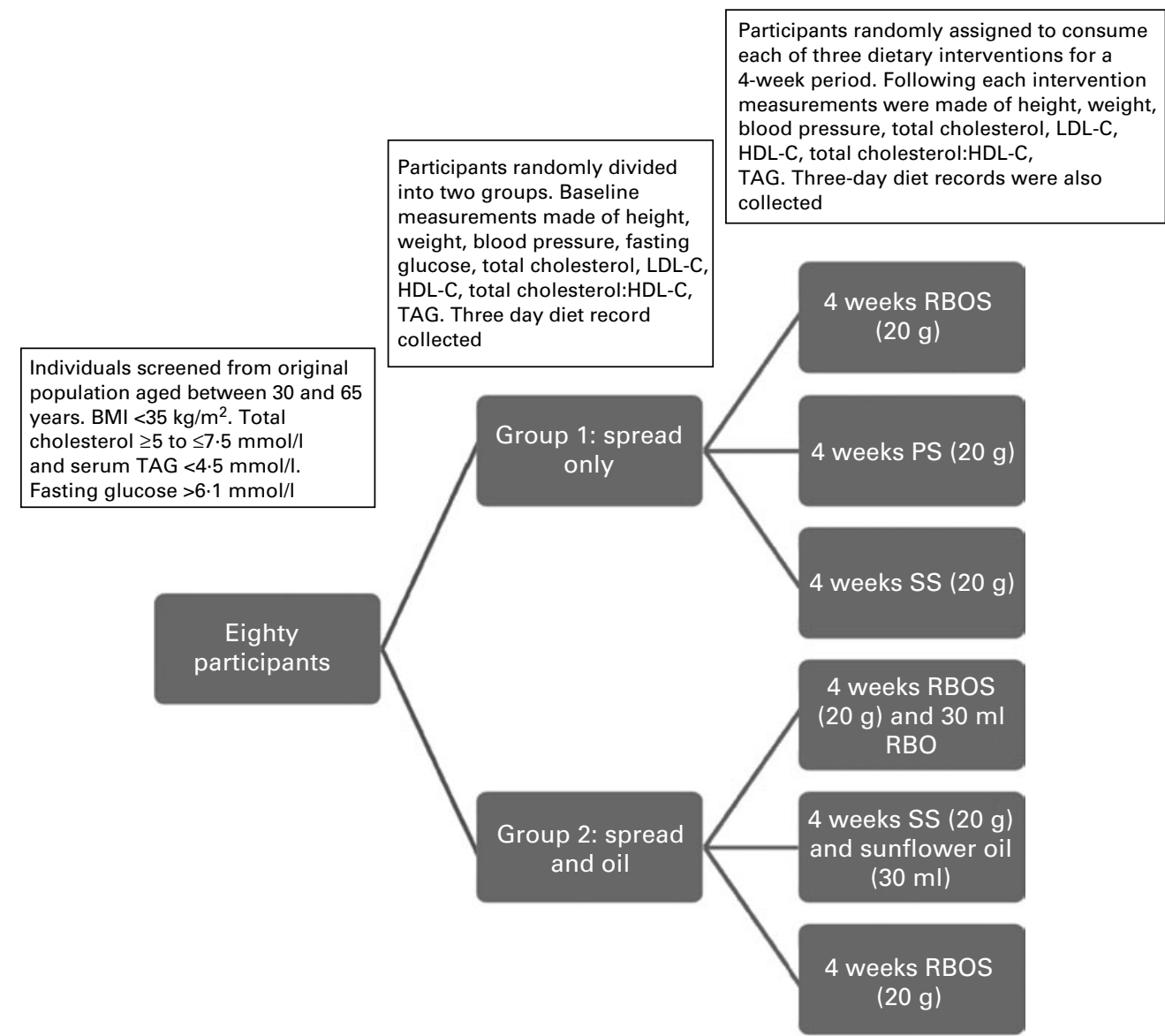

Fig. 1. Schematic of the trial design. LDL-C, LDL-cholesterol; HDL-C, HDL-cholesterol; RBOS, rice bran oil spread; PS, phytosterol-enriched spread; SS, standard spread. 
Table 1. Compositional data of spread (per $100 \mathrm{~g})^{\star}$

\begin{tabular}{lcll}
\hline & RBOS & $\begin{array}{l}\text { Standard } \\
\text { spread }\end{array}$ & $\begin{array}{l}\text { Phytosterol-enriched } \\
\text { spread }\end{array}$ \\
\hline Energy (kJ) & 3010 & 2600 & 2380 \\
Protein (g) & 0.5 & $<1$ & $<1$ \\
Fat, total (g) & 81.0 & 70 & 64 \\
SFA (g) & 23.0 & $18(\max )$ & $15(\max )$ \\
PUFA (g) & $24 \cdot 8$ & $30(\min )$ & $26(\min )$ \\
MUFA (g) & $32 \cdot 0$ & $17(\min )$ & $19(\min )$ \\
Oryzanol (g) & 1.5 & 16 & \\
Vitamin E (mg) & 432 & 16 & \\
$n-3$ ALA (g) & & 2 min & \\
$n-6$ (g) & & 28 min & \\
Cholesterol (mg) & & $<3$ & 8 \\
Plant sterols (g) & & & \\
\hline
\end{tabular}

RBOS, rice bran oil spread, max, maximum; min, minimum; ALA, $\alpha$-linolenic acid * Data supplied by the manufacturers.

LDL-C was only calculated if the TAG measurement was less than $4.5 \mathrm{mmol} / 1$ (Canterbury Health Laboratories).

The results presented for lipid analysis are the mean values of the two individual measurements taken $48 \mathrm{~h}$ apart. Blood glucose measurements were obtained via an enzymatic method using glucose hexokinase and an Aeroset/c8000 analyser (Abbott Clinical Chemistry, Auckland, New Zealand).

\section{Diet record analysis}

Dietary intake was assessed by estimated diet records completed on three non-consecutive days (including one weekend/non-working day). Detailed instructions for filling out the record were given to each participant (the records and photographic assessment records were purchased from the Department of Human Nutrition, Otago University, Dunedin, New Zealand). Records were completed at baseline and at the end of the first, second and third intervention periods. Nutrient intakes were analysed using the New Zealand database of Foodworks professional, version 4, 2005 (Xyris Software, Highgate Hill, QLD, Australia) based on the 1999 New Zealand food composition data from the New Zealand Institute for Plant and Food Research Limited (Christchurch, New Zealand) ${ }^{(18)}$.

\section{Statistical analysis}

Standard descriptive statistics, including means and standard deviations, were used to summarise plasma and diet data for the entire cohort of seventy-five individuals who completed the study (see below), and separately for the two groups of thirty-nine (group 1) and thirty-six (group 2). A total sample of forty in each group in this cross-over design enabled effect sizes of $>0.45$ to be detected as statistically significant (two-tailed $\alpha=0.05$ ) with $80 \%$ power. Comparisons of the three cross-over interventions were undertaken within each of the two groups and utilised repeated-measures ANOVA to test for statistical significance. Where significant treatment effects were detected, planned pairwise comparisons were undertaken between treatments (post hoc analysis). The appropriateness of the parametric ANOVA models was confirmed by visual inspection of residual plots from the models to confirm normality. Data were also analysed for any effect arising from the order in which participants received each treatment.

\section{Results}

Of the eighty individuals who initially agreed to participate, seventy-five completed the 12 -week study. The five individuals who withdrew from the study were two males and three females: three developed illness unrelated to the study product and two had an unforeseen change in circumstance. No adverse effects related to the treatment spread were noted over the course of the study. Compliance with consuming the trial products was reported to be greater than $90 \%$ as assessed by self-completed check sheets. Table 3 demonstrates the baseline characteristics of the study population.

No significant differences were detected in BMI, with the exception of results from the treatment of RBO plus RBOS, which produced a reduction of $0 \cdot 22$ from baseline (26.83-26.61) achieving a low level of significance $(P=0.04)$. Additionally, no significant differences were detected in systolic or diastolic blood pressure throughout the study.

Changes in serum lipoprotein status were observed for participants in the spread-only group when consuming RBOS and PS. Both products produced significant changes in TC, TC:HDL-C and LDL-C levels. No changes were observed for HDL-C or TAG. The results are presented in Table 4.

Within group 1 , RBOS reduced TC by $2 \cdot 2 \%$ compared with a $4 \cdot 1 \%$ reduction with PS. However, RBOS produced a greater reduction $(4.1 \%)$ in the TC:HDL ratio $(P=0.005)$ than the PS $(3.4 \%, P=0.01)$, in addition to reducing LDL-C by $3.5 \%$ compared with SS. PS produced a $5.6 \%$ reduction in LDL-C compared with SS within the spread-only group.

In group 2, the effect of consuming additional amounts of the corresponding base oil to the spread was tested on the basis that increasing the intake of plant sterols would increase the reduction in cholesterol. However, in this group, we did not observe any reductions in any cholesterol levels (Table 5).

Table 2. Compositional data of oil (per $100 \mathrm{~g})^{*}$

\begin{tabular}{|c|c|c|}
\hline & RBO & Sunflower oil \\
\hline Energy (kJ) & 3700 & 3760 \\
\hline Protein (g) & 0.0 & Trace \\
\hline Fat, total (g) & 100 & 99 \\
\hline $\operatorname{SFA}(g)$ & $19 \cdot 9$ & $11.7 \mathrm{~g}$ \\
\hline PUFA (g) & $32 \cdot 1$ & 61.9 \\
\hline $\operatorname{MUFA}(\mathrm{g})$ & $40 \cdot 6$ & $21 \cdot 1$ \\
\hline Cholesterol & $0.0 \dagger$ & $0 \ddagger$ \\
\hline $\mathrm{Na}(\mathrm{g})$ & 0.0 & Trace \\
\hline Trans-fatty acids (g) & 0.0 & \\
\hline Vitamin E (mg) & 25 & \\
\hline$\alpha$-Tocopherol (mg) & $5-20$ & \\
\hline Tocotrienols (mg) & $20-50$ & \\
\hline Oryzanol (mg) & 500 & \\
\hline Phytosterol (mg) & 740 & \\
\hline
\end{tabular}


Table 3. Baseline characteristics of the study participants (Mean values and standard deviations)

\begin{tabular}{|c|c|c|c|c|c|c|}
\hline \multirow[b]{2}{*}{ Variables } & \multicolumn{2}{|c|}{$\begin{array}{l}\text { Total study } \\
\text { group }(n 80)\end{array}$} & \multicolumn{2}{|c|}{$\begin{array}{l}\text { Group 1: } \\
\text { spread only } \\
\quad(n 40)\end{array}$} & \multicolumn{2}{|c|}{$\begin{array}{l}\text { Group 2: } \\
\text { spread plus oil } \\
(n 40)\end{array}$} \\
\hline & Mean & SD & Mean & SD & Mean & SD \\
\hline \multicolumn{7}{|l|}{$\operatorname{Sex}(n)$} \\
\hline Male & \multirow{2}{*}{\multicolumn{2}{|c|}{$\begin{array}{l}27 \\
53\end{array}$}} & \multirow{2}{*}{\multicolumn{2}{|c|}{$\begin{array}{l}10 \\
30\end{array}$}} & \multirow{2}{*}{\multicolumn{2}{|c|}{$\begin{array}{l}17 \\
23\end{array}$}} \\
\hline Female & & & & & & \\
\hline Age (years) & $52 \cdot 1$ & $7 \cdot 9$ & 51.5 & $8 \cdot 1$ & $52 \cdot 8$ & $7 \cdot 6$ \\
\hline Weight (kg) & $72 \cdot 9$ & $14 \cdot 8$ & $70 \cdot 7$ & 13.9 & $75 \cdot 2$ & $15 \cdot 6$ \\
\hline BMI $\left(\mathrm{kg} / \mathrm{m}^{2}\right)$ & $25 \cdot 8$ & 3.9 & 24.9 & 3.5 & $26 \cdot 6$ & 4.0 \\
\hline Total cholesterol (mmol/l) & $5 \cdot 9$ & 0.7 & $5 \cdot 8$ & 0.7 & 5.9 & 0.8 \\
\hline HDL-cholesterol (mmol/l) & 1.5 & 0.3 & 1.5 & 0.3 & 1.4 & $0 \cdot 3$ \\
\hline LDL-cholesterol (mmol/l) & $3 \cdot 8$ & 0.7 & $3 \cdot 8$ & 0.6 & $3 \cdot 8$ & $0 \cdot 8$ \\
\hline Total cholesterol:HDL-cholesterol & 4.2 & 1.1 & 4.0 & 0.9 & 4.4 & 1.2 \\
\hline TAG (mmol/l) & $1 \cdot 3$ & 0.5 & $1 \cdot 2$ & 0.5 & $1 \cdot 3$ & 0.6 \\
\hline Systolic blood pressure $(\mathrm{mmHg})$ & 128.5 & $19 \cdot 3$ & $128 \cdot 2$ & $19 \cdot 5$ & $128 \cdot 8$ & $19 \cdot 3$ \\
\hline Diastolic blood pressure $(\mathrm{mmHg})$ & $77 \cdot 7$ & $10 \cdot 5$ & $77 \cdot 2$ & $10 \cdot 9$ & $78 \cdot 2$ & $10 \cdot 0$ \\
\hline
\end{tabular}

\section{Dietary information}

Participants were asked to complete four $3 \mathrm{~d}$ diet records over the course of the trial, one at baseline and one after each treatment. Characteristics of the dietary variables from these diet records are shown in Tables 6 and 7 .

\section{Dietary intake differences from baseline dietary intake and between treatments}

Dietary records were analysed for an average of the total energy consumed over each $3 \mathrm{~d}$ period recorded in each of the three treatment phases in each section of the trial. Average intakes of each macronutrient over the $3 \mathrm{~d}$ period were then calculated as a percentage of the total energy.

\section{Total energy}

There were no significant differences from baseline dietary intake (defined as dietary intake before beginning the dietary intervention) detected in total energy intake for participants when consuming RBOS ( $P=0 \cdot 422)$. However, when consuming PS and SS, total energy intake decreased significantly by $9.3 \%(P=0.008)$ and $10 \%(P=0.003)$, respectively. In group 2 , the effect of substituting habitual spread intake with the trial spread products plus the corresponding oil showed varying responses. The treatment of SS plus sunflower oil resulted in no significant changes from baseline $(P=0.089)$, but there was a significant increase of $10 \%(P<0 \cdot 001)$ in total energy observed during the treatment of RBOS plus RBO. During the intervention stages, there were no significant changes in

Table 4. Spread-only group*

(Mean values, standard deviations and differences, $n$ 39)

\begin{tabular}{|c|c|c|c|c|c|c|}
\hline & \multicolumn{6}{|c|}{ Spread-only group } \\
\hline & Treatment & Mean & SD & $\begin{array}{c}\text { Paired differences } \\
\text { (adjusted for baseline) }\end{array}$ & Difference (\%) & $P \dagger$ \\
\hline \multirow[t]{3}{*}{ Cholesterol } & SS $v$. RBOS & $5 \cdot 8-5 \cdot 7$ & $0.6,0.6$ & 0.13 & $2 \cdot 2$ & 0.045 \\
\hline & SS v. PS & $5 \cdot 8-5 \cdot 5$ & $0.6,0.6$ & 0.24 & $4 \cdot 1$ & 0.001 \\
\hline & RBOS $v$. PS & $5 \cdot 7-5 \cdot 5$ & $0.5,0.6$ & 0.11 & 1.9 & 0.095 \\
\hline \multirow{3}{*}{ TAG } & SS $v$. RBOS & $1 \cdot 2-1 \cdot 3$ & $0.5,0.9$ & -0.11 & -0.08 & 0.405 \\
\hline & SS $v$. PS & $1 \cdot 2-1 \cdot 2$ & $0.5,0.5$ & -0.01 & -0.82 & 0.781 \\
\hline & PS $v$. RBOS & $1 \cdot 2-1 \cdot 3$ & $0.5,0.9$ & -0.1 & -7.5 & 0.481 \\
\hline \multirow[t]{3}{*}{ HDL-C } & SS $v$. RBOS & $1.4-1.5$ & $0.3,0.3$ & -0.02 & $-1 \cdot 32$ & 0.45 \\
\hline & SS $v . P S$ & $1.5-1.5$ & $0.3,0.3$ & 0.00 & 0 & 0.76 \\
\hline & PS $v$. RBOS & $1.4-1.5$ & $0.33,0.31$ & -0.02 & $-1 \cdot 32$ & 0.27 \\
\hline \multirow[t]{3}{*}{ Total cholesterol:HDL } & SS $v$. RBOS & $4 \cdot 1-3 \cdot 9$ & $1 \cdot 1,0.9$ & 0.17 & $4 \cdot 2$ & 0.005 \\
\hline & SS v. PS & $4 \cdot 1-3.9$ & $1 \cdot 1,1 \cdot 1$ & 0.14 & 3.4 & 0.014 \\
\hline & PS $v$. RBOS & $3.9-3.9$ & $1 \cdot 1,0.9$ & 0.02 & 0.51 & $0.64 c$ \\
\hline \multirow[t]{3}{*}{ LDL-C } & SS $v$. RBOS & $3.7-3.6$ & $0.6,0.5$ & 0.13 & 3.5 & 0.016 \\
\hline & SS $v$. PS & $3 \cdot 7-3.5$ & $0.6,0.6$ & 0.21 & $5 \cdot 6$ & 0.001 \\
\hline & PS $v$. RBOS & $3.5-3.6$ & $0.6,0.5$ & -0.08 & 0.02 & 0.245 \\
\hline
\end{tabular}

SS, standard spread; RBOS, rice bran oil spread; PS, phytosterol-enriched spread; HDL-C, HDL-cholesterol; LDL-C, LDL-cholesterol.

${ }^{*}$ Changes to lipid parameters; comparison of SS with RBO and PS treatments.

$\dagger P \leq 0.05$ (ANOVA). 
Table 5. Spread plus oil group*

(Mean values, standard deviations and difference, $n$ 39)

\begin{tabular}{|c|c|c|c|c|c|}
\hline & \multicolumn{5}{|c|}{ Spread plus oil group } \\
\hline & Treatment & Mean & SD & $\begin{array}{l}\text { Paired differences } \\
\text { (adjusted for baseline) }\end{array}$ & $P \dagger$ \\
\hline \multirow[t]{3}{*}{ Total cholesterol } & RBOS $v$. RBOS and RBO & $5 \cdot 9-5 \cdot 8$ & $0.8,0.6$ & 0.07 & 0.323 \\
\hline & RBOS $v$. SS plus sunflower oil & $5 \cdot 9-5 \cdot 8$ & $0.8,0.8$ & 0.08 & 0.352 \\
\hline & RBOS and RBO $v$. SS and sunflower oil & $5 \cdot 8-5 \cdot 8$ & $0.8,0.8$ & 0.01 & 0.973 \\
\hline \multirow[t]{3}{*}{ TAG } & RBOS v. RBOS and RBO & $1.4-1.4$ & $0.7,0.6$ & 0.08 & 0.21 \\
\hline & RBOS $v$. SS and sunflower oil & $1.4-1.4$ & $0.7,0.6$ & 0.08 & 0.13 \\
\hline & RBOS and RBO v. SS and sunflower oil & $1.4-1.4$ & $0.6,0.6$ & 0 & 0.99 \\
\hline \multirow[t]{3}{*}{ HDL-C } & RBOS v. RBOS and RBO & $1.5-1.5$ & $0.3,0.4$ & -0.01 & 0.69 \\
\hline & RBOS $v$. SS and sunflower oil & $1.5-1.5$ & $0.3,0.3$ & -0.02 & 0.35 \\
\hline & RBOS and RBO v. SS and sunflower oil & $1.5-1.5$ & $0.4,0.3$ & -0.01 & 0.66 \\
\hline \multirow[t]{3}{*}{ Total cholesterol:HDL } & RBOS v. RBOS and RBO & $4 \cdot 3-4 \cdot 3$ & $1 \cdot 2,1 \cdot 2$ & 0.02 & 0.69 \\
\hline & RBOS $v$. SS and sunflower oil & $4 \cdot 3-4.2$ & $1 \cdot 2,1 \cdot 2$ & 0.10 & 0.09 \\
\hline & RBOS and RBO v. SS and sunflower oil & $4 \cdot 3-4 \cdot 2$ & $1 \cdot 2,1 \cdot 1$ & 0.08 & 0.32 \\
\hline \multirow[t]{3}{*}{ LDL-C } & RBOS $v$. RBOS and RBO & $3 \cdot 8-3 \cdot 8$ & $0 \cdot 7,0.8$ & -0.03 & 0.79 \\
\hline & RBOS $v$. SS and sunflower oil & $3.8-3.8$ & $0.6,0.7$ & 0.01 & 0.81 \\
\hline & RBOS and RBO v. SS and sunflower oil & $3.8-3.8$ & $0.8,0.7$ & 0.04 & 0.65 \\
\hline
\end{tabular}

RBOS, rice bran oil spread; RBO, rice bran oil; SS, standard spread; HDL-C, HDL-cholesterol; LDL-C, LDL-cholesterol.

${ }^{*}$ Changes to lipid parameters; comparison of RBOS plus RBO to standard spread with sunflower oil plus RBOS treatments.

$\dagger P<0.05$ (ANOVA).

total energy as a result of any of the treatments except between the RBOS treatment section and the RBOS plus RBO treatment section. The RBOS plus RBO treatment section resulted in the participants showing a significant increase in total energy of $8.2 \%(P=0.004)$ from that observed with RBOS.

\section{Carbohydrate as a percentage of total energy}

No significant changes were observed from baseline in carbohydrate intake during the RBOS $(P=0.284)$, PS $(P=0.795)$, SS $(P=0.162)$ or RBOS plus RBO $(P=0.685)$ treatment. A significant decrease in the carbohydrate intake as a percentage of energy of $11.5 \%(P<0.001)$ from baseline was observed during the treatment with SS plus sunflower oil. There were no significant differences detected in carbohydrate intake between the treatment sections, except for between the RBOS plus RBO treatment and the SS plus sunflower oil treatment, which resulted in a decrease in the carbohydrate intake of $9.8 \%(P=0.04)$.

\section{Protein as a percentage of total energy}

No significant changes were observed from baseline during the RBOS $(P=0.93)$, PS $(P=0.287)$ or SS $(P=0.405)$ treatment. Significant decreases in protein intake as a percentage of energy of $11.0 \%(P=0.008)$ were observed with the SS plus sunflower oil treatment, and of that $14.6 \%(P<0.001)$ with the RBOS plus RBO treatment. Protein intake remained constant between all treatments, except between treatment with the RBOS and the RBOS plus RBO treatment, where a decrease of $6 \%(P=0.01)$ was demonstrated.

\section{Total fat intake as a percentage of total energy}

Significant changes from baseline were not observed during any of the treatment phases in the spread-only group, although a trend towards an increase in total fat was demonstrated (RBO, $P=0 \cdot 07$; PS, $P=0 \cdot 07$; SS, $P=0 \cdot 08$ ). For all other treatments, fat intake as a percentage of energy increased significantly: SS and sunflower oil resulted in an increase of $13.6 \%(P<0 \cdot 001)$, and RBOS plus RBO treatment resulted in

Table 6. Energy and nutrient composition as a percentage of total energy of dietary intake: group 1 - spread only group (Mean values and standard deviations)

\begin{tabular}{|c|c|c|c|c|c|c|c|c|}
\hline & \multicolumn{2}{|c|}{ Baseline } & \multicolumn{2}{|c|}{ RBOS } & \multicolumn{2}{|c|}{ Phytosterol spread } & \multicolumn{2}{|c|}{ Standard spread } \\
\hline & Mean & SD & Mean & SD & Mean & SD & Mean & SD \\
\hline Energy (kJ) & 8735 & 1724 & 8330 & 1909 & $7927(\downarrow)^{\star \star}$ & 1409 & $7861(\downarrow)^{\star \star}$ & 1741 \\
\hline $\mathrm{CHO}(\% \mathrm{TE})$ & 49 & 7 & 47 & 7 & 48 & 10 & 47 & 7 \\
\hline Protein (\%TE) & 17 & 3 & 18 & 3 & 18 & 4 & 18 & 3 \\
\hline Fat (\%TE) & 31 & 7 & 34 & 7 & 34 & 9 & 33 & 8 \\
\hline SFA (\%TE) & 12 & 4 & 11 & 3 & 12 & 4 & 12 & 4 \\
\hline MUFA (\%TE) & 11 & 2 & 11 & 3 & $13 \uparrow^{\star \star *}$ & 2 & 11 & 3 \\
\hline PUFA (\%TE) & 5 & 2 & $7 \uparrow^{\star \star \star}$ & 2 & $7 \uparrow^{\star \star \star}$ & 2 & $8 \uparrow^{* \star *}$ & 2 \\
\hline Cholesterol (mg) & 264 & $10 \overline{3}$ & $206 \downarrow^{* \star}$ & 93 & 243 & 110 & $208 \downarrow^{* \star}$ & $11 \overline{1}$ \\
\hline
\end{tabular}

RBOS, rice bran oil spread; $\mathrm{CHO}$, carbohydrate, $\downarrow$, decrease; \%TE, percentage of total energy; $\uparrow$, increase. Mean values were significantly different: ${ }^{\star \star} P \leq 0.01,{ }^{\star \star \star} P \leq 0.001$. 
Table 7. Energy and nutrient composition as a percentage of total energy of dietary intake: group 2 - spread plus oil

(Mean values, standard deviations and difference, $n$ 36)

\begin{tabular}{|c|c|c|c|c|c|c|c|c|}
\hline & \multicolumn{2}{|c|}{ Baseline } & \multicolumn{2}{|c|}{ RBOS and oil } & \multicolumn{2}{|c|}{$\begin{array}{l}\text { Standard spread } \\
\text { and sunflower oil }\end{array}$} & \multicolumn{2}{|c|}{ RBOS } \\
\hline & Mean & SD & Mean & SD & Mean & SD & Mean & SD \\
\hline Energy (kJ) & 8189 & 207 & $8983 \uparrow$ * & 178 & 8766 & 174 & 8241 & 194 \\
\hline $\mathrm{CHO}$ (\%TE) & 52 & 8 & 51 & 3 & $46 \downarrow^{* \star *}$ & 7 & 51 & 7 \\
\hline Protein (\%TE) & 17 & 3 & $15 \downarrow^{* \star *}$ & 3 & $15 \downarrow^{\star * *}$ & 4 & 16 & 4 \\
\hline Fat (\%TE) & 32 & 9 & $40 \uparrow^{* \star \star}$ & 6 & $37 \uparrow^{* *}$ & 7 & 34 & 6 \\
\hline SFA (\%TE) & 13 & 5 & 12 & 3 & 12 & 5 & 12 & 3 \\
\hline MUFA (\%TE) & 11 & 3 & $15 \uparrow$ *** & 3 & $15 \uparrow * *$ & 8 & 12 & 2 \\
\hline PUFA (\%TE) & 6 & 2 & $9 \uparrow^{* * *}$ & 2 & $9 \uparrow * \star \star$ & 5 & $8 \uparrow * * *$ & 3 \\
\hline Cholesterol (mg) & 217 & 104 & 208 & 93 & 225 & 86 & $193 \downarrow^{\star \star}$ & 84 \\
\hline
\end{tabular}

RBOS, rice bran oil spread; $\mathrm{CHO}$, carbohydrate; $\uparrow$, increase; \%TE, percentage of total energy; $\downarrow$, decrease Mean values were significantly different: ${ }^{\star} P \leq 0.05$, ${ }^{\star \star} P \leq 0.01,{ }^{\star \star \star} P \leq 0.001$.

an increase of $20 \%(P<0 \cdot 001)$. Between treatments, an increase of $14.3 \%(P=0.002)$ was observed between treatment with the RBOS and the RBOS plus RBO treatment. No significant differences were detected as a result of any of the other treatments.

\section{Saturated fat intake as a percentage of total energy}

Treatment with RBOS, PS and SS did not result in any significant changes in saturated fat intake as a percentage of energy from baseline ( $P=0.24,0.087$ and 0.73 , respectively). Additionally in group 2 , no significant differences from baseline were observed (RBOS plus RBO, $P=0 \cdot 37$; SS plus sunflower oil, $P=0.36$; RBOS, $P=0.34$ ). The saturated fat content of the diets also remained constant in all treatments.

\section{Monounsaturated fat intake as a percentage of total energy}

Treatment with PS resulted in a significant increase of $15 \%$ in monounsaturated fat $(P<0 \cdot 001)$. Similarly, rises of $15 \%$ were shown with RBOS and RBO $(P<0.001)$ and SS and sunflower oil $(P<0 \cdot 01)$. Between treatment arms, a significant increase of $20 \%(P<0.001)$ was observed during the RBOS and RBO and SS and sunflower oil phases when compared with the RBOS only.

\section{Polyunsaturated fat intake as a percentage of total energy}

Significant increases from baseline were observed in all treatments for polyunsaturated fat intake as a percentage of total energy: RBOS of $27 \%$ ( $P \leq 0.001)$; PS of $21 \%(P<0.001)$; SS of $28 \%(P<0 \cdot 001)$; SS plus sunflower oil of $39 \%(P<0.001)$ and RBOS plus RBO treatment of $38 \%(P<0 \cdot 001)$. Between treatments, no significant differences were observed.

\section{Dietary cholesterol intake}

No significant changes from baseline were observed in the PS treatment $(P=0 \cdot 248)$, SS plus sunflower oil treatment
( $P=0.646)$ or RBOS plus RBO treatment $(P=0.644)$ in dietary cholesterol intake. Significant decreases in dietary cholesterol intake $(\mathrm{mg} / \mathrm{d})$ were observed in the RBOS treatment of $22 \%$ $(P<0 \cdot 01)$ and SS treatment of $21 \%(P<0.01)$. Between treatments, a significant decrease of $14.2 \%(P=0.04)$ was observed from the SS plus sunflower oil treatment to the RBOS. No other differences between treatments were detected.

\section{Discussion}

The present study demonstrated that, compared with a SS, RBOS containing $1.5 \%$ plant sterols reduced TC $(2.2 \%)$, LDL-C (3.5\%) and the TC:HDL-C ratio ( $4.1 \%)$ when consumed at a level of $20 \mathrm{~g}$ (four teaspoons)/d as part of the daily diet over 4 weeks in mildly hypercholesterolaemic subjects. None of the spreads affected HDL-C or TAG concentrations. Daily consumption of $20 \mathrm{~g}$ PS containing plant sterols at a level of $8 \%$ demonstrated a greater reduction in TC (4.4\%) and LDL-C (5.6\%), but was observed to contain $81 \%$ more phytosterol than RBOS. This suggests that while consumption of RBOS did not result in as much lowering of LDL-C when consumed on a weight-for-weight basis as PS, it still provided a significant reduction, which may be of clinical relevance when it is considered that a $1 \%$ reduction in LDL-C can result in a $2 \%$ reduction in $\mathrm{CHD}^{(19)}$. When the relative amounts of plant sterols provided by RBOS are taken into consideration (RBOS, $118 \mathrm{mg}$ phytosterol and $30 \mathrm{mg} \gamma$-oryzanol; PS, $1600 \mathrm{mg}$ phytosterol), it appears that consuming the RBO product resulted in a greater proportional reduction than PS. This may be due to the other portions of the unsaponified fraction found only in RBO. The presence of $\gamma$-oryzanol, tocotrienols and tocopherols and other minor constituents such as squalene and phenolic compounds, in addition to plant sterols, has been shown to elicit strong hypercholesterolaemic properties in several studies ${ }^{(20)}$, and it is possible that these extra components had some effect on the reduction in serum cholesterol parameters observed in this section of the trial. Furthermore, the ability of RBOS to reduce the TC:HDL concentrations to a greater level than the PS ( $4.2 v .3 .4 \%)$ may be indicative of further health benefits that can be 
attributed to this product. Elevated plasma TAG levels are a significant independent risk factor for the development of CHD and are also inversely correlated with HDL-C levels. A high TAG:HDL ratio also correlates with LDL phenotype B, small HDL particles and insulin resistance, making it an important component of the metabolic syndrome and thus a factor in morbidity and mortality ${ }^{(21,22)}$. The ability of RBOS to reduce this ratio demonstrates that the regular consumption of products enriched with plant sterols may affect other lipid variables in addition to LDL-C lowering that requires clarification with long-term randomised, placebo-controlled trials.

Recent studies involving plant sterols ${ }^{(23,24)}$ have suggested that low doses may be effective in lowering LDL-C. Racette et $a l{ }^{(23)}$ demonstrated a dose-dependent effect of plant sterols using a placebo-controlled clinical study to show that while doses of up to $2000 \mathrm{mg} / \mathrm{d}$ produced a significant reduction in LDL-C, moderate doses of plant sterols $(459 \mathrm{mg} / \mathrm{d})$ can also result in the reduction of serum LDL-C of up to $5 \%$, and that these doses are obtainable using a healthy diet without supplementation. Additionally, Escurriol et al. ${ }^{(24)}$ showed that the addition of $158 \mathrm{mg} / \mathrm{d}$ plant sterols to individuals consuming the Mediterranean diet by supplementation with nuts can result in significant reductions $(8.4 \%)$ of LDL-C. The present study supports the fact that effective reductions of LDL-C can be achieved with much smaller plant sterol intakes (approximately $150 \mathrm{mg} / \mathrm{d}$ ), which are similar to doses already consumed by individuals living in Western populations as part of their typical daily intake, suggesting that products derived from RBO may provide an option for lowering the levels of supplementation from the $2 \mathrm{~g} / \mathrm{d}$ advised by the National Cholesterol Education Programme while still providing an effective reduction in serum cholesterol parameters. As an intervention strategy, a lower dose of plant sterol supplementation would not be recommended for individuals with higher LDL-C levels, as it is evident that doses of $2 \mathrm{~g} / \mathrm{d}$ provide optimal LDL-C-lowering protection. It may, however, provide a significantly cost-effective method for those individuals with mildly elevated LDL-C levels, not only since $\mathrm{RBO}$ is produced as a by-product of rice milling and is therefore relatively cheap to produce, but also because phytosterol supplementation as a therapy for reduction of CHD is significantly less expensive than traditional pharmacological treatments ${ }^{(25,26)}$. The addition of higher levels of plant sterols to food products affects its retail price. RBO, with its innate plant sterols, provides the manufacturers and thus consumers with an effective, lower-cost and alternative source of phytosterol that can provide a long-term nonpharmacological treatment strategy for the reduction of cholesterol when included in daily food intake. Additionally, the vitamin $\mathrm{E}$ isomers and $\gamma$-oryzanol also present in the RBOS may confer additional health benefits, particularly antioxidant activity, which has been credited to these compounds in several studies ${ }^{(19,27-29)}$. Further research to identify other health benefits of RBO would be beneficial, for example, studies examining the possible anti-inflammatory action of $\gamma$-oryzanol on markers of CHD risk and possible antioxidant properties exerted by the vitamin $\mathrm{E}$ isomers present in the unsaponified fraction.
The results of this trial are consistent with those shown by other studies investigating the effect of plant sterols on cholesterol. Numerous human intervention trials have reported that a daily consumption of $1.5-3 \mathrm{~g}$ of plant sterols/stanol can reduce TC by $8-15 \%^{(4,9,11)}$. The mechanism of action of plant sterols in products such as the PS used in this trial is not fully understood, but is based on the inhibition of the absorption of dietary and endogenously produced cholesterol because of the similarity in physical structure borne by plant sterols ${ }^{(19)}$. This similarity allows plant sterols to compete with cholesterol for space in the mixed micelle, which has limited capacity, resulting in less cholesterol being absorbed. Plant sterols may also reduce the esterification rate of cholesterol in the enterocyte and thus the amount of cholesterol excreted via the chylomicrons, or limit transmembrane transport by their presence in the unstirred water layer or other mucosal barriers ${ }^{(30-32)}$

The cholesterol-lowering effects observed in the present study were independent of the diet normally consumed by the participants, as demonstrated in previous studies ${ }^{(33,34)}$, suggesting that the effects of these products may be greater if consumed as part of a healthy diet, low in saturated fat and cholesterol and high in vegetables, fruits and whole grains ${ }^{(35)}$.

In addition to the group of participants consuming spread only, the second section of this trial included a group of individuals consuming RBOS along with RBO. It was hypothesised that the increase in the intake of plant sterols $(340 \mathrm{mg} / \mathrm{d}$ as opposed to $118 \mathrm{mg} / \mathrm{d}$ ) may further increase the cholesterollowering effect. There were no significant changes in any serum lipid parameters for any of the participants in this part of the study. The RBOS alone did not replicate the reduction in cholesterol parameters that was demonstrated in the spread-only section of the trial. As yet, we have no satisfactory explanation for this.

It is possible that the addition of RBO to the RBOS may not have been effective because of the higher level of dietary fat provided by the addition of the oil to the diet. It is well documented that high levels of SFA in the diet can increase serum total and LDL-cholesterol ${ }^{(36,37)}$. However, examination of the dietary information generated from these participants did not support this theory. The dietary intake data indicated that during the study, participants' total dietary fat intakes were about $34 \%$ for the spread-only group. Significant increases were observed during the treatment period in the SS plus sunflower oil treatment group $(P \leq 0 \cdot 01)$ and the RBOS plus RBO treatment group $(P \leq 0 \cdot 001)$. However, closer examination of the intakes of individual fatty acids showed that the levels of saturated fat intake decreased compared with baseline values taken before the intervention, and the rise in total fat was attributable to a significant increase in monounsaturated and polyunsaturated fat, both of which are reported to have favourable effects on LDL-C profiles ${ }^{(38)}$. The significant increase in PUFA may have been expected to contribute to the reductions of LDL-C, as rigorous clinical testing of these fatty acids has shown that PUFA (especially linoleic acid, 18:2) are potent total and LDL-C-lowering nutrients, although they demonstrate little effect on HDL-C levels ${ }^{(39)}$. Similarly, 
other studies $^{(40)}$ have shown that MUFA are also effective at lowering plasma LDL-C, although these fatty acids do not appear to be as effective at lowering TAG levels. In the present study, however, it appears that the LDL-C-lowering effect can be attributed to the phytosterol content of the RBOS and PS, as consumption of the sunflower spread alone did not result in a reduction of plasma LDL-C despite having greater PUFA content than both the RBOS and PS. There may have been a greater effect from these fatty acids if the reduction of SFA in the diet had been greater, as their lipid-lowering potential is observed in studies where the PUFA and MUFA content have been used to replace the SFA content of the diet.

Potentially, it is also possible that any effect of an increase in total energy and fat intake during the spread and oil treatment of the trial may have been negated by physiological changes during the digestive process. Dietary fat entering the small intestine results in the stimulation of gut peptides, which control the satiety and food intake. The peptide cholecystokinin signals the gall bladder to produce bile, resulting in the emulsification of fat into micelles and subsequent digestion via lipase enzymes from the pancreas and small intestine ${ }^{(41)}$. It may therefore be possible that the higher levels of dietary fat intake were compensated for by an increase in bile production and fat emulsification contributing to the null findings from the RBOS and RBO. Additionally, although there were some significant differences between macronutrient intakes during some of the different interventions, there were no apparent trends that would imply that the nutrient content of the background diet was responsible for the lack of a cholesterol-lowering effect. Compliance of the participants in consuming the products in this part of the study may also have been underestimated, as the volume of spread and oil supplied for consumption was reported by the participants to be much more than those individuals would normally eat. The reliance on self-reporting as a measure of compliance is a major limitation of the present study.

The way the oil products were used may also have been a contributing factor to the lack of a hypercholesterolaemic effect. Although some individuals reported consuming the oil as a stand-alone product, a majority used the oil for cooking. Studies have shown that although heat treatment may only confer a modest reduction in the levels of plant sterols, the concentration of phytosterol oxides increases ${ }^{(42)}$. These oxidative products may interact with lipids, carbohydrates and proteins present in food matrices that may mask small changes in lipid parameters, and thus have a subsequent effect on phytosterol stability, absorption and health attributes. Further research in this area is required to evaluate the effects of phytosterol oxides ${ }^{(42)}$.

Participants in the present study were randomly assigned using a simple randomisation technique by the statistician to the spread-only group or the spread plus oil group. While it appears from Table 3 that the treatment groups were similar in terms of all relevant participant characteristics (particularly lipoprotein profiles), the present study may have benefited from using a stratified randomisation to ensure that there was no possibility of any characteristic influencing response to treatment. A major proportion of the population group were female (fifty-three $v$. twenty-seven). Differences in lipoprotein metabolism between the sexes are well documented, with women showing a faster rate of fat transport in the blood stream and greater responses to high-fat/high-carbohydrate diets in terms of TAG response and lack of LDL-C lowering, which ultimately is thought to translate to a greater risk of CVD for women linked to these abnormalities ${ }^{(43,44)}$. However, studies examining the differences in sex-specific responses to individual fatty acids have shown that female and male subjects show similar plasma lipid responses to dietary fat ${ }^{(45)}$ and, furthermore, sex differences in plasma cholesterol-lowering response to phytosterol feeding have not been reported in the literature ${ }^{(46,47)}$. The sex imbalance among the participants in the present study was therefore not thought to be detrimental to the results of the study, although it is acknowledged that this area is unexplored and is possibly a very important area of investigation.

The study may have benefited from the inclusion of a washout period between treatments. Washout periods are included to remove any possible carry-over effects from the first treatment period into the second treatment. We decided not to include a washout period between treatment sections, as the length of each treatment was 4 weeks, which provided enough time for the stabilisation of lipid levels even after a change in dietary intake. Previous studies ${ }^{(48,49)}$ have shown that plasma lipid levels stabilise within 2-3 weeks after dietary changes are made. Additionally, with respect to increasing systemic levels of plant sterols that may have resulted from a lack of washout, a recent study ${ }^{(50)}$ has shown that high daily intakes of plant sterols $(8.8 \mathrm{~g} / \mathrm{d})$ result in comparable low levels in serum to doses of $2-3 \mathrm{~g} / \mathrm{d}$ and that the levels are normalised within 4 weeks, suggesting that high doses of these compounds do not result in increased long-term systemic availability. Nguyen ${ }^{(51)}$ has also shown that changes are rapid following the initiation of plant sterol usage into the diet, and maximum changes occur after 2 weeks. It is acknowledged, however, that the exact mechanism of action of plant sterols is not yet fully understood and that in addition to the effect on mixed micellar composition, changes have also been observed at a genetic level through the activation of liver X receptor-regulated genes in the liver ${ }^{(52)}$. Thus, any long-term effects from changes at the genetic level were not accounted for in the present study, and possible effects induced by the prolonged exposure to plant sterols via this mechanism add to the limitations of the present study. The inclusion of a washout period in the present study would have increased the length of the study and may have led to lowered participant compliance and a greater number of withdrawals from the study, which may also have adversely affected the results.

A further complicating issue relates to the variation in blood cholesterol concentrations that occurs daily within individuals, ranging from 5 to $10 \%$ as a consequence of a number of factors, including dietary intake, alcohol consumption, menstrual cycle fluctuations, hydration status, illness and physical 
activity $^{(53)}$. While every effort was made to standardise the collection of blood samples and account for these factors (taking two fasting samples with at least $24 \mathrm{~h}$ in between, at the same time of day, and monitoring dietary intake through dietary intake records, and changes in activity levels through face-to-face interviews during clinic visits), the participants in the present study were individuals and their exact behaviours cannot be itemised. Natural day-to-day variation in blood lipid parameters may have had an impact when examining small changes.

\section{Conclusion}

Non-pharmacological therapies for lowering serum LDLcholesterol levels are an essential part of reducing the risk of $\mathrm{CHD}$, which remains a leading cause of morbidity and mortality worldwide. The addition of foods fortified with plant sterols into the daily diet can significantly reduce cholesterol levels independently of background dietary intakes, and the introduction of these foods has offered another dimension for the dietary management of cholesterol. Consumption of RBOS significantly reduced TC, LDL-cholesterol and total:HDL-C compared with consumption of SS. PS produced a greater reduction in the cholesterol parameters measured but is observed to contain much higher concentrations of plant sterols. RBOS has therefore been shown to be an effective alternative method for reducing lipid parameters, presenting the consumer with a more economical choice of plant sterol-based spread. Additionally, this product may have further as-yet unidentified health benefits arising from its other bioactive compounds ( $\gamma$-oryzanol, tocotrienol and tocopherols), found in the unsaponified fraction. This aspect of $\mathrm{RBO}$ products requires more investigation.

Further research is required to explain the lack of a hypercholesterolaemic effect when RBOS was consumed in conjunction with the RBO. The higher amounts of plant sterols consumed by individuals when combining test products did not affect cholesterol parameters, suggesting that there may be some effects resulting from the method of cooking or unreported compliance issues that need addressing. However, the results of the present study, supported by other investigations into the cholesterol-lowering effect of plant sterol and stanol products, provide significant evidence that these compounds are an effective and safe adjunct to diet-controlled therapy, which can produce clinically significant reductions in LDL-cholesterol in people with mild to moderate hypercholesterolaemia.

\section{Acknowledgements}

The present study was funded by the Hansells Food Group, Auckland, New Zealand. The authors have no conflicts of interest to declare. S. E., A. W., C. F., R. S. and J. W. were involved in the study design. S. E. and J. W. were involved in the data collection; S. E. and A. W. were involved in the data analysis; C. F. carried out the statistical analysis. S. E., A. W. and J. W. wrote the manuscript.

\section{References}

1. National Heart, Lung and Blood Institute (NHLBI) (2002) National Cholesterol Education Programme (National Cholesterol Education Programme) Expert Panel on Detection, Evaluation and Treatment of High Blood Cholesterol in Adults (Adult Treatment Panel III) final report. Circulation 106, 3143-3421.

2. Executive summary of The Third Report of the National Cholesterol Education Programme (National Cholesterol Education Programme) (2001) Expert Panel on Detection, Evaluation, and Treatment of High Blood Cholesterol in Adults (Adult Treatment Panel III). JAMA 285, 2486-2497.

3. Plat J \& Mensink RP (2005) Plant stanol and sterol esters in the control of blood cholesterol levels. Mechanism and safety aspects. Am J Cardiol 96, 15D-22D.

4. Law M (2000) Plant sterol and stanol spreads and health. BMJ 320, 861-864.

5. Piironen V, Lindsay DG \& Miettienen TA (2000) Plant sterols: biosynthesis, biological function and their importance to human nutrition. J Sci Food Agric 80, 939-966.

6. Devaraj S \& Jialal I (2006) The role of dietary supplementation with plant sterols and stanols in the prevention of cardiovascular disease. Nutr Rev 647, 348-354.

7. Fernandes P \& Cabral JMS (2006) Plant sterols: applications and recovery methods. Bioresour Technol. http://www. eslevier.com

8. Salo P \& Wester I (2005) Low fat formulations of plant stanols and sterols. Am J Cardiol 96, 51D-54D.

9. Katan MB, Grundy SM, Jones P, et al. (2003) Efficacy and safety of plant stanols and sterols in the management of blood cholesterol levels. Mayo Clin Proc 78, 965-978.

10. St-Onge MP \& Jones PJH (2003) Plant sterols and human lipid metabolism: efficacy, safety and novel foods. Lipids 384, 367-375.

11. AbuMweis SS, Barake R \& Jones PJH (2008) Plant sterols/ stanols as cholesterol lowering agents: a meta-analysis of randomised controlled trials. Food Nutr Res 52. http:// www.foodandnutritionresearch.net

12. Qureshi AA, Sami SA \& Khan FA (2002) Effects of stabilized rice bran, its soluble and fibre fractions on blood glucose levels and serum lipid parameters in humans with diabetes mellitus types I and II. J Nutr Biochem 13, 175-187.

13. Wilson TA, Nicolosi RJ, Woolfrey B, et al. (2007) Rice bran oil and oryzanol reduce plasma lipid and lipoprotein cholesterol concentrations and aortic cholesterol ester accumulation to a greater extent than ferulic acid in hypercholesterolemic hamsters. J Nutr Biochem 18, 105-112.

14. Lee J-W, Lee S-W, Kim M-K, et al. (2005) Beneficial effect of the unsaponifiable matter from rice bran oil on oxidative stress in vitro compared with $\alpha$-tocopherol. J Sci Food Agric 85, 493-498.

15. Most MM, Tulley R, Morales S, et al. (2005) Rice bran oil, not fibre, lowers cholesterol in humans. Am J Clin Nutr 81, 64-68.

16. Cicero AFG \& Gaddi A (2001) Rice bran oil and $\gamma$-oryzanol in the treatment of hyperlipoproteinaemias and other conditions. Phyto Res 15, 227-289.

17. Friedweld WT, Levy RT \& Fredrickson DS (1972) Estimation of the concentration of low-density lipoprotein cholesterol in plasma, without use of the preparative ultracentrifuge. Clin Chem 18, 499-502.

18. Lesperence L (2009) The Concise New Zealand Food Composition Tables, 8th ed. Wellington/Palmerston North: New Zealand Institute for Plant and Food Research/Ministry 
of Health. http://www.crop.cri.nz/home/products.services/ nutrition/foodcompsitiondata/consise8ed.pdf

19. Ostlund RE (2007) Plant sterols, cholesterol absorption and healthy diets. Lipids $\mathbf{4 2}, 41-45$.

20. De Deckere EAM \& Korver O (1996) Minor constituents of rice bran oil as functional foods. Nutr Rev 11, s120-s133.

21. Jeppesen J, Hein HO, Suadicani P, et al. (1997) Relation of high TG-low HDL cholesterol and LDL cholesterol to the incidence of ischemic heart disease: an eight year follow up in the Copenhagen Male Study. Arterioscler Thromb Vasc Biol 17, 1114-1120.

22. Derdemezis CS, Filippatos TD, Mikhailidis DP, et al. (2010) Review Article: effects of plant sterols and stanols beyond low density lipoprotein cholesterol lowering. J Cardiovasc Pharmacol Ther 15, 120-134.

23. Racette SB, Lin X, Lefevre M, et al. (2010) Dose effects of dietary phytosterols on cholesterol metabolism: a controlled feeding study. Am J Clin Nutr 91, 32-38.

24. Escurriol V, Cofan M, Serra M, et al. (2009) Serum sterol responses to increasing plant sterol intake from natural foods in the Mediterranean diet. Eur J Nutr 48, 373-382.

25. Gerber A, Evers T, Haverkamp H, et al. (2006) Cost-benefit analysis of a plant sterol containing low fat spread for cholesterol reduction. Eur J Health Econom 7, 247-254.

26. Wiboonsirikul J, Kimura Y, Kanaya Y, et al. (2008) Production and characterisation of functional substances from a by-product of rice bran oil and protein production by compressed hot water treatment. Biosci Biotechnol Biochem 722, 384-392.

27. Sugano M \& Tsuji E (1997) Rice bran oil and cholesterol metabolism. J Nutr 127, 521-524.

28. Godber JS, Xu Z, Hegsted M, et al. (2002) Rice bran and rice bran oil in functional foods development. Louisiana Agricultural Magazine 454, 9-10.

29. Jariwalla RJ (2001) Rice bran products: phytonutrients with potential applications in preventative and clinical medicine. Drugs Exp Clin Res 27, 17-26.

30. Lichtenstein AH \& Deckelbaum RJ (2001) Stanol/sterol ester containing foods and blood cholesterol levels. Circulation 103, 1177-1179.

31. De Jong N, Zuur A, Wolfs MC, et al. (2007) Exposure and effectiveness of phytosterol/stanol-enriched spreads. Eur $J$ Clin Nutr 61, 1407-1415.

32. Ostlund RE (2002) Plant sterols in human nutrition. Ann Rev Nutr 22, 533-549.

33. Westrate J \& Meijer GW (1998) Plant sterol enriched spreads and reduction of plasma total and LDL cholesterol concentrations in normocholesterolemic and mildly hypercholesterolaemic subjects. Eur J Clin Nutr $\mathbf{5 2}$, $334-343$.

34. Hendricks HF, Westrate JA, van Vliet T, et al. (1999) Spreads enriched with three different levels of vegetable oil sterols and the degree of cholesterol lowering in normocholesterolaemic and mildly hypercholesterolaemic subjects. Eur J Clin Nutr 534, 319-327.

35. Maki C, Davidson MH, Umporowicz DM, et al. (2001) Lipid responses to plant-sterol enriched reduced fat spreads incorporated into a National Cholesterol Education Program Step 1 diet. Am J Clin Nutr 74, 33-43.
36. Mensink RP \& Katan MB (1992) Effect of dietary fatty acids on serum lipids and lipoproteins. A meta-analysis of 27 trials. Arterioscler Thromb 12, 911-919.

37. Hu FB, Manson JE \& Willet WC (2001) Types of dietary fat and risk of coronary heart disease: a critical review. I Am Coll Nutr 20, 5-19.

38. Gibson RS (2005) Measuring food consumption of individuals. In Principles of Nutritional Assessment, pp. 41-50. Oxford: Oxford University Press.

39. Sacks FM \& Campos H (2006) Polyunsaturated fatty acids, inflammation and cardiovascular disease: time to widen our view of the mechanisms. J Clin Endocrinol Metab 91, 398-400.

40. Gardner CD \& Kraemer HC (1995) Monosaturated versus polyunsaturated dietary fat and serum lipids: a meta analysis. Arterioscler Thromb Vasc Biol 15, 1917-1927.

41. Beglinger C \& Degen L (2004) Fat in the intestine as a regulator of appetite - role of CCK. Physiol Behav $\mathbf{8 3}$, 617-621.

42. Soupas L, Juntunen L, Lampi AM, et al. (2004) Effects of sterol structure, temperature and lipid medium on phytosterol oxidation. J Agric Food Chem 52, 6485-6491.

43. Knopp RH, Paramsothy P, Retzlaff BM, et al. (2005) Gender differences in lipoprotein metabolism and dietary response: basis in hormonal differences and implications for cardiovascular disease. Curr Atheroscler Rep 7 , 472-479.

44. Knopp RH, Paramsothy P, Retzlaff BM, et al. (2006) Sex differences in lipoprotein metabolism and dietary response: basis in hormonal differences and implications for cardiovascular disease. Curr Atheroscler Rep 8, 452-459.

45. Lapointe A, Balk EM \& Lictehnstein AH (2006) Gender differences in plasma lipid response to dietary fat. Nutr Rev $\mathbf{6 4}$, 234-249.

46. Jones PJH, MacDougall DE, Ntanios F, et al. (1997) Dietary phytosterols as cholesterol lowering agents in humans. Can J Physiol Pharmacol 75, 217-227.

47. Micallef MA \& Manohar L (2008) The lipid lowering effects of phytosterols and (n-3) polyunsaturated fatty acids are synergistic and complementary in hypolipidaemic men and women. J Nutr 138, 1086-1090.

48. Bonanome A \& Grundy SM (1988) Effect of dietary stearic acid on plasma on cholesterol and lipoprotein levels. $N$ Engl J Med 318, 1244-1248.

49. O’Dea K, Traianedes K, Chisholm K, et al. (1990) Cholesterol lowering effect of a low fat diet containing both lean beef is reversed by the addition of beef fat. Am JClin Nutr 52, 491-494.

50. Gylling H, Hallikainen M, Nissinen MJ, et al. (2010) Very high plant stanol intake and serum plant stanols and non-cholesterol sterols. Euro J Nutr 49, 111-117.

51. Nguyen TT (1999) The cholesterol lowering action of plant stanol esters. J Nutr 129, 2109-2112.

52. Plat J, Nichols JA \& Mensink RP (2005) Plant Sterols and stanols: effects on mixed micellar composition and LXR (target gene) activation. J Lipid Res 46, 2468-2476.

53. Grandjean PW \& Alhassan S (2006) Essential laboratory methods for blood lipid and lipoprotein analysis. In Lipid Metabolism and Health, pp. 117-142 [RJ Moffat and B Stamford, editors]. Boca Raton, FL: CRC Press. 